\title{
Evaluación de la calidad de vida en mujeres con vaginismo primario mediante el WHOQOL-BREF
}

\author{
Andrés Moltedo-Perfetti ${ }^{a}$, María José Cittadinia , Bernardo Nardi ${ }^{b}$, Emidio Arimatea $^{2 a}$, \\ Piero Moltedo-Perfetti. ${ }^{\mathrm{C}}$ \\ ${ }^{1}$ Pontificia Universidad Católica de Valparaíso, Chile. ${ }^{2}$ Centro Adolescenti-Clinica Psichiatrica-Dipartimento di Medicina \\ Clinica e Sperimentale, Università Politecnica delle Marche, Ancona, Italia. ${ }^{3}$ Universidad Santo Tomás, Chile.
}

$\mathrm{a}_{\text {Psicólogo/a. }}{ }^{\mathrm{b}}$ Psiquiatra. ${ }^{\mathrm{c}}$ Ingeniero Comercial.

\section{RESUMEN}

Antecedentes: El vaginismo es una disfunción sexual que impide la penetración y que afecta a cerca de un $5 \%$ de las mujeres y que les genera malestar tanto a ellas como a sus parejas. Objetivo: Conocer la calidad de vida de mujeres con vaginismo primario y comparar con un grupo control. Método: Aplicación presencial del WHOQOL-BREF a 50 mujeres con vaginismo primario y a 47 mujeres sin patología, sociodemográficamente similares. Resultados: El nivel de calidad de vida en las mujeres con vaginismo fue menor a aquella del grupo de comparación respecto a la calidad general de la vida y la salud, y en tres de Ios cuatro dominios del WHOQOL-BREF. Conclusión: El vaginismo es una condición que afecta la calidad de vida de las mujeres con ese diagnóstico.

\section{PALABRAS CLAVES: Vaginismo, disfunción sexual, calidad de vida, WHOQOL-BREF}

\section{SUMMARY}

Background: Vaginismus is a sexual dysfunction that prevents the penetration and affects about $5 \%$ of women and makes them feel upset both them and their partners. Objective: Determine the quality of life of women with primary vaginismus and compared with a control group. Method: Application of the WHOQOLBREF to 50 women with primary vaginismus and 47 women without pathology and similar demographically status. Results: The level of quality of life in women with vaginismus was lower than that of the comparison group regarding the overall quality of life and health, and in three of the four domains of the WHOQOLBREF. Conclusion: Vaginismus is a condition that affects the quality of life of women with this diagnosis.

\section{KEY WORDS: Vaginismus, sexual dysfunction, quality of life, WHOQOL-BREF}

\section{INTRODUCCIÓN}

El vaginismo puede ser definido como una contracción muscular involuntaria del primer tercio de la vagina (tercio externo) que impide la penetración y la realización del coito (1) a pesar del deseo y del grado de excitación de la mujer. Se produce en el momento que la mujer anticipa que se producirá la penetración, siendo un espasmo condicionado por la fobia a la penetración, generalmente asociada cognitivamente al dolor (2).

La prevalencia oscila entre un 2 y un $4 \%$ de las mujeres adultas $(3,4)$, aunque algunos investigadores llegan a referir hasta un $7 \%$ de las mismas (5). 
Su incidencia en el número de consultas por disfunciones sexuales oscila entre un 5 y $20 \%(6-10)$, pero es altamente probable que se encuentre subreportada debido a lo sensible del trastorno y a la vergüenza generalmente asociada a este problema $(5,11,12,13)$.

Los criterios para su diagnóstico por parte del DSM IV-TR (14) son los siguientes: A) Aparición persistente o recurrente de espasmos involuntarios de la musculatura del tercio externo de la vagina, que interfiere el coito. B) La alteración provoca malestar acusado o dificultad en las relaciones interpersonales. C) El trastorno no se explica mejor por la presencia de otro trastorno mental (por ejemplo, trastorno de somatización) y no es debido exclusivamente a los efectos fisiológicos directos de una enfermedad médica. De acuerdo a su intensidad se puede clasificar como leve, moderado o severo (15) y acorde a su duración y origen como primario o de toda la vida y secundario o adquirido (14).

Al momento de consultar con especialistas pueden llevar años de actividades sexuales no coitales en pareja, pero generalmente acuden debido a sentimientos de culpa ante la imposibilidad de la penetración o de quedar embarazadas, situaciones que les acarrean dificultades a nivel de pareja o sociales por no tener descendencia $(3,16)$.

Como se aprecia, para establecer el diagnóstico resulta necesario que las dificultades en el funcionamiento sexual ocurran de manera persistente, causen malestar psicológico en la persona y/o que genere problemas en la pareja $(14,17)$, por lo que se hipotetiza que una mujer con este diagnóstico vería afectada su calidad de vida (CV).

La CV es un concepto polisémico (18), cuyo estudio científico en las Ciencias Sociales en general y en la Psicología en particular, ha tenido un desarrollo exponencial en las últimas décadas (19). Su origen se puede remontar a los Estados Unidos tras la Segunda Guerra Mundial y su uso se expande a mediados de los años sesenta (20), cuando los economistas y científicos sociales comienzan a realizar investigaciones en CV recolectando información y datos objetivos como el nivel socioeconómico, educacional o el tipo de vivienda (21). Sin embargo, dichos indicadores muchas veces resultaban insuficientes para explicar la varianza en la calidad de vida individual (22), por lo que algunos autores hipotetizaron que las variables subjetivas, como la felicidad y la satisfacción, serían aquellas que podrían explicar de mejor forma el fenómeno (23).

En un segundo momento, la medición de la calidad de vida ha tenido un enfoque basado en el déficit, centrándose en los problemas de los individuos y sus factores asociados en el ambiente, definiéndola como la ausencia de problemas, en concordancia con la definición de salud de la Organización Mundial de la Salud (OMS) de 1948 que la define como: "un estado de completo bienestar físico, mental y social, que no sólo comporta la ausencia de enfermedad" (24).

Durante las últimas décadas se ha propuesto un cambio en la noción tradicional del concepto de $\mathrm{CV}$, para el cual el bienestar no es ni puede ser reconocido sólo como la ausencia de dificultades, problemas o patologías $(25,26)$, sino más bien vinculándola al bienestar con el contacto social y las relaciones interpersonales (27), con la participación social, arraigo y contactos comunitarios (28), con la familia y el contacto social (29), con los recursos sociales (30), con las relaciones positivas con los demás $(31,32)$ y con el funcionamiento social $(33)$, entre otros.

En la actualidad, la Organización Mundial de la Salud, define CV como "la percepción individual de su posición en la vida en el contexto de su cultura y sistemas de valores en donde vive y la relación hacia sus metas, expectativas, estándar e intereses" (34). De esta definición se desprenden tres ámbitos de la CV: físico, psicológico y social.

Para la OMS la CV resulta importante al considerar las decisiones que pueden tomar los pacientes respecto de sus tratamientos, así como la evaluación de programas y la focalización de recursos (35). Es por ello que surge la necesidad de contar con instrumentos que permitan comparar distintas poblaciones o distintas patologías, para poder realizar comparaciones epidemiológicas, así como la comprensión de la enfermedad y tipos de tratamiento más eficaces (35).

En los años 90, la OMS diseñó un instrumento que permite evaluar la $\mathrm{CV}$ en colaboración con 15 centros de diferentes países para lograr una perspectiva transcultural (36), el WHOQOL-100, el cual, tras una serie de análisis redujo su número de ítems a 26, dando origen al WHOQOL-BREF (37) el cual ya ha sido validado y aplicado en diversos idiomas y países (38-43), como en grupos específicos (44-48), así como en Chile, donde se han reportado sus características psicométricas $(49,50)$ $y$ ha sido utilizado en investigaciones respecto de situaciones específicas (51-54).

Al estudiar las consecuencias del vaginismo en las distintas dimensiones de la CV hay una serie de estudios relacionados, por ejemplo en lo referente a la salud física, estudios encontraron una relación entre mujeres con vaginismo y el dolor (55). En otros, centrados en el bienestar psicológico se encontró que en las mujeres con vaginismo la salud mental era menor que en las mujeres de la población normal, sufriendo en mayor medida de depresión, menor autoestima y dificultades cognitivas (56). De la misma manera, como ya se ha dicho con anterioridad (57), el miedo, la ansiedad, el disgusto y el asco han sido señalados como factores predisponentes y precipitantes importantes en la bibliografía respecto del vaginismo (58-61). 
En lo referente al ámbito social, las investigaciones se han centrado principalmente en los efectos del vaginismo en la relación de pareja, mientras que en una serie de investigaciones se ha señalado que las mujeres con vaginismo presentaban una menor calidad de la relación de pareja $(4,8,62,63)$, en tanto que en otros se señala que las mujeres con vaginismo no presentaban diferencias significativas con las demás mujeres respecto de su nivel de satisfacción con su relación de pareja $(64,65)$.

Sin embargo, la mayoría de los estudios respecto del vaginismo se centran en dimensiones aisladas o particulares de la CV de las mujeres con vaginismo, faltando estudios que traten acerca de la globalidad del impacto en la CV de dichas mujeres, tal como lo define la OMS y se evalúa a través del WHOQOL, con la sola excepción de un estudio realizado en Países Bajos (66).

Es por lo anterior que el objetivo del estudio fue ver cómo se afectaba la CV de un grupo de mujeres adultas chilenas que asisten a terapia psicológica, diagnosticadas con vaginismo primario, sin antecedentes de alteraciones físicas o ginecológicas y con una historia de vida sin traumas ni abusos sexuales, en comparación con un grupo similar de mujeres sin patología, utilizando el WHOQOL-BREF.

\section{PACIENTES Y MÉTODO}

Se seleccionó a 50 mujeres que asistieron a consulta psicológica-sexológica por motivo de vaginismo primario, y como grupo control a 47 mujeres elegidas de manera pareada al grupo estudio en las variables edad, estudios, estado civil y nivel socioeconómico. Todas ellas mujeres mayores de edad y que participaron de manera voluntaria.

El cuestionario contenía preguntas socio-demográficas y la versión chilena del WHOQOL-BREF. El instrumento está compuesto por 26 preguntas, de las cuales una de ellas apunta a saber sobre la CV general, otra sobre satisfacción con la salud y las 24 restantes se agrupan en 4 dominios de la CV (salud física, 7 preguntas; salud psicológica, 6 preguntas; relaciones sociales, 3 preguntas; y ambiente, 8 preguntas). Las escalas de respuesta son de tipo Likert con 5 opciones, donde las encuestadas debían responder a cada pregunta en categorías entre 1 y 5 puntos y los puntajes obtenidos en los distintos dominios fueron posteriormente convertidos acorde con una tabla de corrección producto de la desigualdad en el número de ítems, ya sea en un rango de 0 a 100 o de 4 a 20 (67). En este caso, se ha optado por la primera opción. Se considera que a mayor puntuación obtenida, mayor CV y bajas puntuaciones corresponden a una CV baja.

Este instrumento ha sido validado y adaptado semánticamente en Chile con una consistencia interna de $0,89(49)$ y 0,88 (50) para la escala total y un rango de 0,63 y 0,76 (49) y 0,7 a 0,79 (50) para cada una de las dimensiones.

Al grupo control, además de la aplicación del cuestionario, se le realizó una entrevista clínica por parte de un psicoterapeuta acreditado, con más de 15 años de experiencia clínica, para descartar la presencia de alguna psicopatología.

Análisis de los datos: La comparación entre el grupo con vaginismo primario y el grupo control en lo relacionado con las variables socio-demográficas fue realizada mediante la prueba de ji cuadrado. En lo referente de las puntuaciones del WHOQOL se utilizó la T de Student. Se utilizó el programa SPSS versión 19.

\section{RESULTADOS}

Características socio-demográficas de los participantes. Las variables sociodemográficas descritas en la Tabla I no mostraron diferencias significativas entre el grupo de las participantes con vaginismo y el grupo control, en términos de edad, escolaridad y estatus marital. La mayoría tenía un alto nivel de educación y una relación estable.

Tabla I

CARACTERÍSTICAS SOCIO-DEMOGRÁFICAS DE LOS PARTICIPANTES

\begin{tabular}{lccc}
\hline Variable & $\begin{array}{c}\text { Vaginismo } \\
(\mathrm{n}=50)\end{array}$ & $\begin{array}{c}\text { Control } \\
(\mathrm{n}=47)\end{array}$ & $\begin{array}{c}\text { Valor } \mathrm{p} \\
{ }^{*} x^{2}\end{array}$ \\
\hline Edad \pm DE & $27,65 \pm 8,16$ & $27,80 \pm 9,18$ & ${ }^{*} 0,50$ \\
$18-25$ años & $34(68 \%)$ & $32(68,09 \%)$ & - \\
$26-35$ años & $15(30 \%)$ & $14(29,78 \%)$ & - \\
$\geq 36$ años & $1(2 \%)$ & $1(2,13 \%)$ & - \\
Casada/conviviendo & 27 & 33 & ${ }^{*} 0,79$ \\
Años de estudio & $13,60 \pm 3,71$ & $13,45 \pm 3,28$ & ${ }^{*} 0,71$ \\
\hline
\end{tabular}


La calidad de vida de acuerdo con el WHOQOL$B R E F$. En este estudio se encontró una consistencia interna de 0,86 para la escala total y un rango de 0,65 y 0,77 entre cada una de las dimensiones. En la Tabla II se muestran las puntuaciones medias de las encuestadas en el WHOQOL-BREF. Se puede apreciar que, de acuerdo a los resultados de este instrumento, existen diferencias en los niveles de CV entre ambos grupos, donde las mujeres con vaginismo presentan un menor nivel de $\mathrm{CV}$ en los dominios "calidad general de la vida y la salud", "salud física", "relaciones sociales" y "ambiente", mientras que en "salud psicológica" no se apreciaron diferencias significativas.

\section{Tabla II \\ LA CALIDAD DE VIDA DE ACUERDO CON EL WHOQOL-BREF}

\begin{tabular}{lccc}
\hline $\begin{array}{l}\text { WHOQOL-BREF } \\
\text { Dominio* }\end{array}$ & $\begin{array}{c}\text { Vaginismo } \\
M \pm D E\end{array}$ & $\begin{array}{c}\text { Control } \\
M \pm D E\end{array}$ & Valor P \\
\hline $\begin{array}{l}\text { Calidad general de } \\
\text { la vida y la salud }\end{array}$ & $7,4 \pm 1,2$ & $8,1 \pm 0,9$ & $<0,01$ \\
Salud física & $63,3 \pm 4,6$ & $76,2 \pm 5,2$ & $<0,01$ \\
$\begin{array}{l}\text { Salud psicológica } \\
\text { Relaciones }\end{array}$ & $61,8 \pm 5,7$ & $64,1 \pm 4,2$ & $\mathrm{NS}$ \\
sociales & $54,9 \pm 4,8$ & $70,1 \pm 5,5$ & $<0,01$ \\
Ambiente & $68,3 \pm 4,3$ & $75,2 \pm 3,8$ & $<0,01$ \\
\hline
\end{tabular}

*El rango de puntuación de la calidad de vida general es 2-10.

Las puntuaciones de cada dominio van de 0-100.

NS: no significativo.

\section{DISCUSIÓN}

Los resultados de esta investigación muestran que el vaginismo tiene consecuencias negativas para la CV de las mujeres que lo presentan. Estos efectos negativos se extienden más allá de las relaciones sociales o de pareja, ya que se aprecian valores menores en tres de los cuatro ámbitos de la WHOQOL-BREF, así como en la pregunta referente a la "calidad general de la vida y la salud".

Desde un punto de vista clínico, junto con la imposibilidad de quedar embarazada (recibiendo presiones sociales al respecto, especialmente en aquellas mujeres casadas), uno de los principales motivos de consulta de las mujeres con este diagnóstico apunta a las "relaciones sociales", presentando insatisfacción con su vida sexual, tanto por parte de sí mismas o producto de la presión de su pareja por tener relaciones coitales, lo que se tra- duce en dificultades de tipo relacional. Tal como se señalaba, si bien existe en la bibliografía evidencia contradictoria respecto del impacto del vaginismo en la CV, los resultados de esta investigación apuntan a señalar que, efectivamente, tal como lo señalan algunos $(4,8,62,63)$ las mujeres con vaginismo presentan diferencias significativas en el nivel de satisfacción de su relación de pareja (menor CV), impacto que será mayor o menor al interior de cada pareja específica, dependiendo de factores idiosincráticos.

En lo referente a la "salud física", tal como se ha expresado en investigaciones anteriores (57), el temor al dolor y a la dependencia es un aspecto esencial en este trastorno $(10,13)$, al punto de que el vaginismo puede ser considerado como una suerte de fobia o un miedo real, producto ya sea de un condicionamiento o de una experiencia anticipada ante la penetración $(3,8,57)$.

Como se ha mencionado anteriormente, si bien es común que muchas mujeres con vaginismo presenten sentimientos negativos hacia sí mismas o se sientan en deuda con sus parejas y familias (desde un punto de vista sexual o reproductivo), el impacto en los indicadores de salud psicológica no es significativo. Una explicación de aquello radica en la posible negación o evitación de la disfunción sexual debido al temor fóbico al dolor o al miedo a perder el control (57). Sin embargo, también es posible que las mujeres con vaginismo, por factores relacionados con su educación no le den la importancia a las relaciones sexuales que sí les dan otras mujeres.

A pesar de los resultados antes señalados, hay que mencionar que esta menor $\mathrm{CV}$ reflejada en las puntuaciones en el WHOQOL podría no ser producto del impacto del vaginismo en sí, sino producto de cualquier patología sexual, por lo que habría que realizar estudios comparativos con mujeres con otras disfunciones sexuales para referir el real impacto del vaginismo en la CV.

Además, se seleccionaron encuestadas con vaginismo primario sin trauma, no incluyendo secundario o con traumas. Aquellas mujeres con vaginismo secundario pueden tener patrones psicológicos distintos a las mujeres de este estudio, ya que por una parte pueden recibir una menor presión social para tener hijos (ya que algunas pueden haber sido madres), pero a la vez pueden tener parejas que no las comprendan por la aparición de este trastorno y, por tanto, recibir menos apoyo o comprensión de sus parejas, ya que la ausencia de relaciones sexuales con penetración no forman parte de la constitución inicial de la pareja, como sí lo es en el caso de las parejas de este estudio.

En ese mismo sentido, se trata de mujeres que asistieron a consulta privada, por lo que pueden tener más recursos (económicos, intelectuales o autorreferenciales), mayor presión o la noción de que 
hay algo que deben de cambiar en sus vidas (o que pueden estar y sentirse de una manera distinta).

\section{CONCLUSIÓN}

Los resultados de este estudio deben ser utilizados e interpretados con precaución, ya que si bien son concordantes con los obtenidos en otras investigaciones (66), el impacto específico del vaginismo en la CV no está claro y puede variar si se utilizan otros instrumentos de medición de la CV.

\section{REFERENCIAS}

1. Basson R, Berman J, Burnett A, Derogatis L, Ferguson $\mathrm{D}$, Fourcroy $\mathrm{J}$, et al. Report of the international consensus development conference on female sexual dysfunction: Definitions and classifications. J Urol 2000;163:888-93.

2. Basson R. Human sex-response cycles. J Sex Marital Ther 2001;27:33-43.

3. Masters W, Johnson V, Kolodny R. La Sexualidad Humana. Grijalbo. 7a edición, 1987.

4. Hurtado F, Teodoro E, Royo A, Muñoz Y. Estudio sobre disfunciones sexuales femeninas: Incidencia por sexos y análisis de variables biopsicosociales predictoras. Cuadernos de Medicina Psicosomática y Psiquiatría de Enlace 1996;37:24-33.

5. Pacik P. Vaginismus: Review of current concepts and treatment using botox injections, bupivacaine injections, and progresive dilation with the patient under anesthesia. Aesthetic Plast Surg 2001;35:1160-4.

6. Mears E. Sexual problems clinics. An assessment of the work of 26 doctors trained by the Institute of ppsychosexual Medicine. London. Public Health London 1978;92:218-23.

7. Bancroft J. Human sexuality and its problems. Churchill Livingstone. Nueva York, 1983.

8. Heisler J. Sexual therapy in the national marriage guidance council. Marriage Guidance Council, Rugby, 1983.

9. Spector I, Carey M. Incidence and prevalence of the sexual dysfunctions: a critical review of the empirical literature. Arch Sex Behav 1990;19:389-96.

10. Lahaie M, Boyer S, Amsel R, Khalife S, Binik Y. Vaginismus: a review of the literature on classification/ diagnosis etiology, and treatment. Woman's Health 2010;6:705-19.

11. Oniz A, Keskinoglu P, Bezircioglu I. The prevalence and causes of sexual problems among premenopausal Turkish women. J Sex Med 2007;4:1575-81.

12. Nusbaum M, Gamble G, Skinner B, Heiman J. The high prevalence of sexual concerns among women seeking routine gynecological care. J Fam Pract 2000;49:229-32.

13. Reissing $E$, Binik $Y$, Khalife $S$. Does vaginismus exist? A critical review of the literature. J Nerv Ment Dis 1999;187:261-74.
14. American PsyquiatricAssociation. Manual Diagnóstico y Estadístico de los Trastornos Mentales (DSM-IVTR). Madrid: Masson; 2002.

15. Astolfi E. Disfunciones sexuales femeninas: ¿Qué es el vaginismo?; Aprender Salud; HIBA; 2007.

16. Kuile M, Lankveld J, Groot E, Melles R, Neffs J, Zandbergen M. Cognitive-behavioural therapy for women with lifelong vaginismus: Process and prognostic factors. Behav Res Ther 2006;45:359-73.

17. Pacik P. Vaginismus: Review of current concepts and treatment using botox injections, bupivacaine injections, and progresive dilation with the patient under anesthesia. Aesthetic Plast Surg 2001;35:1160-4.

18. Borthwick-Duffy S. Quality of life and quality of care in mental retardation. En: L. Rowitz (Ed). Mental retardation in the year 2000. Berlin: Springer Verlag, 1992. pp $52-66$

19. Bilbao M. Creencias Sociales y Bienestar: Valores, creencias básicas, impacto de los hechos vitales y crecimiento psicológico. Tesis Doctoral, Universidad del País Vasco, 2008.

20. Gómez-Vela M, Verdugo, M. El cuestionario de evaluación de la calidad de vida de alumnos de educación secundaria obligatoria: Descripción, validación inicial y resultados obtenidos tras su aplicación en una muestra de adolescentes con discapacidad y sin ella. Siglocero. Revista Española sobre Discapacidad Intelectual. 2004;35(4)5-17.

21. Urzúa A, Caqueo-Urizar A. Calidad de vida: Una revisión teórica del concepto. Terapia Psicológica 2012; 30(1):61-71.

22. Borgan G. The concept of quality of life. Social and Practice 2005;31:561-80.

23. Haas $B$. Clarification and integration of similar quality of life concepts. J Nursing Scholarships 1999;31:215-20.

24. Organización Mundial de la Salud. Constitución de la Organización Mundial de la Salud (Documento en línea). Disponible en: www.who.int/gb/bd/PDF/bd46/sbd46_p2.pdf. Acceso el 16 de Julio de 2014.

25. Bilbao M, Techio E, Páez D. Bienestar subjetivo, cultura y valores personales: Estado de la cuestión y síntesis metaanalítica de estudios. Revista de Psicología - Edición Especial en Psicología Cultural y Transcultural 2007;25(2):233-76.

26. Blanco A, Díaz D. El bienestar social: su concepto y medición. Psicothema 2005;17(4):582-9.

27. Erikson R. Descripciones de la desigualdad: el enfoque sueco de la investigación sobre el bienestar. En M. Nussbaum y A. Sen (Comps.): La calidad de vida (101-120). México: F.C.E, 1996.

28. Allardt E. Tener, amar, ser: Una alternativa al modelo sueco de investigación sobre el bienestar. En: M. Nussbaum, y A. Sen (Comps.): La calidad de vida. pp 126-134. México: F.C.E, 1996.

29. Diener E. Assessing subjective well-being: Progress and opportunities. Social Indicators Research 1994;31:103-57.

30. Veenhoven R. The four qualities of life. Ordering 
concepts and measures of the good life. $\mathrm{J}$ Happines Studies 2000;1:1-39.

31. Ryff $\mathrm{C}$, Keyes $\mathrm{C}$. The structure of psychological wellbeing revisted. J Personality and Social Psychology 1995; 69 (4):719-27.

32. Keyes C, Ryff C, Shmotkin D. Optimizing well-being: the empirical encounter of two traditions. J Personality and Social Psychology 2002;82:1007-22.

33. Smith K, Avis N, Assmann S. Distinguishing between quality of life and health status in quality of life research: a meta-analysis. Quality of Life Research 1999;8:447-59.

34. WHOQOL Group. The World Health Organization Quality of life assessment (WHOQOL): Position paper from the World Health Organization. Soc Sci Med 1995;41:1403-9.

35. WHOQOL Group. Development of the WHOQOL, Rational and current status. Int $\mathrm{J}$ Ment Health 1994;23(3):24-56.

36. Fleck MP, Louzada S, Xavier M, Chachamovich E, Vieira G, Santos L, Pinzon V. Application of the portuguese version of the instrument for the assessment of the quality of life of the World Health Organization (WHOQOL-100). Rev Saúde Pública 1999;33(2):198205.

37. WHOQOL Group. Development of the World Health Organization WHOQOL-Bref Quality of Life Assessment. Psychol Med 1998;28:551-88.

38. Skevington S, Lotfy M, O'Conell K. The World Health Organization's WHOQOL-Bref quality of life assessment: Psychometric properties and results of the international field trial. A report from the WHOQOL Group. Qual Life Res 2004;13:299-310.

39. Saxena S, Carlson D, Billington R, Orley J. The WHO quality of life assessment instrument (WHOQOLBref): The importance of its items for cross-cultural research. Qual Life Res 2001;10:711-21.

40. Noerholm V, Groenvold M, Watt T, Bjorner J, Rasmussen N, Bech P. Quality of life in the Danish general population-normative data and validity of WHOQOL- Bref using Rasch and item response theory models. Qual Life Res 2004;13:531-40.

41. Moreno A, Faerstein E, Werneck G, Lopes C, Chor D. Propiedades psicométricas do instrumento abreviado de avaliação de qualidade de vida da organização mundial da saúde no estudo pró-saúde. Cad Saúde Pública 2006;22(12):2585-97.

42. Min S, Kim K, Lee C, Jung Y, Suh S, Kim D. Development of the Korean versions of WHO Quality of Life scale and WHOQOL-Bref. Qual Life Res 2002;11:593600

43. Fleck M, Louzada S, Xavier M, Chachamovich E, Vierira I, Santos L, et al. Aplicação da versão em Português do instrumento abreviado de avaliação da qualidade de vida "WHOQOL-Bref". Rev Saúde Pública 2000;34(2):178-83.

44. Trompenaars F, Masthoff E, Van Heck G, Hodiamont $\mathrm{P}$, De Vries J. Content validity, construct validity, and reliability of the WHOQOL-Bref in a population of Dutch adult psychiatric outpatients. Qual Life Res 2005;14:151-60.

45. Fang C, Hsiung P, Yu C, Chen M, Wan J. Validation of the World Health Organization quality of life instrument in patients with HIV infection. Qual Life Res 2002;11:753-62.

46. Yao G, Wu Ch. Factorial Invariance of the WHOQOL- Bref among disease groups. Qual Life Res 2005;14:1881-8.

47. Izutsu T, Tsutsumi A, Islam A, Matsuo $Y$, Sayuri $H$, Kurita $\mathrm{H}$, et al. Validity and realiability of the Bangla version of WHOQOL-Bref on a adolescent population in Bangladesh. Qual Life Res 2005;14:1783-9.

48. Jang $Y$, Hsieh $C$, Wang $Y$, Wu Y. A Validity study of the WHOQoL-Bref Assessment in persons with traumatic spinal cord injury. Arch Phys Med Rehabilitation 2004;85:1890-5.

49. Urzúa A, Caqueo-Urizar A. Estructura Factorial y valores de referencia del WHOQOL-BREF en población adulta chilena. Rev Med Chile 2013;141:1547-54.

50. Espinoza I, Osorio P, Torrejón M, Lucas-Carrasco R, Bunout D. Validación del cuestionario de calidad de vida (WHOQOL-BREF) en adultos mayores chilenos. Rev Med Chile 2011;139:579-86.

51. Urzúa A. Calidad de Vida y Factores Biopsicosociales en Patologías Médicas Crónicas. Ter Psicol 2008;26(2):207-14.

52. Urzúa A, Jarne A. Calidad de vida y estilos de afrontamiento en personas con patologías crónicas. Rev Interam Psicol 2008;42(1):151-60.

53. Torres M, Quezada M, Rioseco R, Ducci ME. Calidad de vida de adultos mayores pobres de viviendas básicas: Estudio comparativo mediante uso de WHOQOL-Bref. Rev Med Chile 2008;136:325-33.

54. Aguirre F. Validación del cuestionario de Calidad de Vida (WHOQOL-BREF) en personas con diagnósti$\cos$ de esquizofrenia y trastornos afines en Chile. Disponible en: www.saludpublica.fcm.unc.edu.ar/sites/ default/files/Validacion\%20del\%20cuestionario\%20 en\%20calidad\%20de\%20vida\%20-\%20WHOQoLBREF.ppt. Acceso el 16 de julio de 2014.

55. De Kruiff M, terKuile M, Weijenborg, $P$, van Lankveld $\mathrm{J}$. Vaginismus and dyspareunia: is there a difference in clinical presentation? J Psychosomatic Obstet Gynaecol 2000;21:149-55.

56. Sánchez C, Carreño J, Corres N, Taracena B. Perfiles e indicadores psicológicos relacionados con la dispareunia y el vaginismo: Estudio cualitativo. Segunda parte. Salud Mental 2010;33(5):437-49.

57. Moltedo A, Nardi B, Arimatea E. Coherencia sistémica e identitaria en mujeres con vaginismo primario. $\mathrm{Re}$ Chil Obstet Ginecol 2014;79(1):56-63.

58. TerKuile M, van Lankveld J, Vlieland C, Willekes C, Weijenborg P. Vulvar vestibulitis syndrome: An important factor in the evaluation of lifelong vaginismus? J Psychosomatic Obstet Gynaecol 2005;26:245-9.

59. Binik Y. The DSM diagnostic criteria for vaginismus. Arch Sex Behav 2010;39:278-91. 
60. Labrador F. Tratamiento psicológico de las disfunciones sexuales. En: Echeburúa, E. (Ed.) Parejas en conflicto: Estrategias psicológicas de intervención. San Sebastián: Universidad del País Vasco, 1987.

61. Bach A, Wincze J, Barlow D. Sexual Dysfunction. En: Barlow, D. (Ed.) Clinical handbook of psychological disorders. New York: The Guilford Press, 2001.

62. Bodenmann G, Ledermann T, Blattner D, Galluzzo C. Association among everyday stress, critical life events and sexual problems. J Nerv Ment Dis 2006;194:494501.

63. Masters W, Johnson V. El Vínculo del Placer. Grijalbo, 14 edición, 1994.

64. Van Lankveld J, Brewaeys A, TerKuile M, Weijenborg $P$. Difficulties in the differential diagnosis of vaginismus, dyspareunia and mixed sexual pain disorder. $\mathrm{J}$ Psychosomatic Obstet Gynaecol 1995;16:201-9.
65. Reissing E, Binik Y, Khalifé S, Cohen D, Amsel R. Etiological correlates of vaginismus: Sexual and physical abuse, sexual knowledge, sexual self-schema and relationship adjustment. J Sex Marital Ther 2003;29:47-59.

66. Rosier A, Drossaert C, de Vries J,Vrij-Hoogendoorn L. De relatie tussen kwaliteit van leven en vaginisme. TvS 2013; 37-1, 1-9.

67. World Health Organization. WHOQOL-Bref: Introduction, administration, scoring and generic version of the assessment. Field Trial Version 1996. Programme on mental health. Geneva. Disponible en: http://www. who.int/mental_health/media/en/76.pdf. Acceso el 16 de Julio de 2014. 